\title{
Modelo empírico entre busca de sensação e as variações do delinquir em brasileiros
}

\section{Empirical model between sensation seeking and the variations of the delinquency in the Brazilian}

\author{
Nilton S. Formiga ${ }^{[a]}$, Catarina Isabel Fernandes Sintra ${ }^{[b]}$, Paulo Lopes ${ }^{[c]}$
}

\footnotetext{
${ }^{[a]}$ Doutor em Psicologia Social pela Universidade Federal da Paraíba, professor no curso de Psicologia na Faculdade Maurício de Nassau, João Pessoa, PB - Brasil, e-mail: nsformiga@yahoo.com

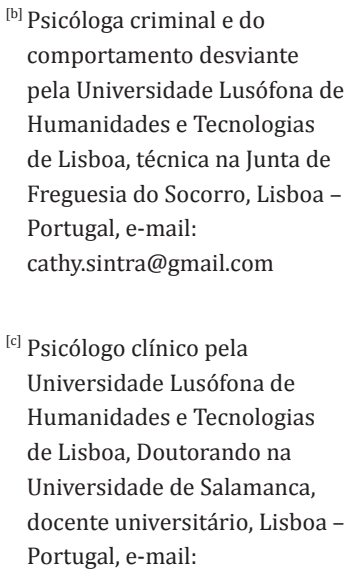

Recebido: 03/05/2012 Received: 05/03/2012

Aprovado: 20/11/2012 Approved: 11/20/2012

\begin{abstract}
Resumo
Apesar de existir muitas variáveis que expliquem o delinquir em jovens, os traços de personalidade ainda tem sido, na Psicologia, um construto muito promissor para compreender esse problema, contribuindo como mais uma resposta na intervenção desse fenômeno. Neste trabalho pretende-se desenvolver, a partir da modelagem de equação estrutural, a explicação da variação do delinquir (composto pelo uso potencial de drogas, condutas desviantes e comportamento agressivo) a partir do construto personalístico da busca de sensação. 284 jovens, de 14 a 20 anos, responderam o questionário do uso potencial de drogas, condutas desviantes, comportamento agressivo e busca de sensação. A partir do programa AMOS GRAFICS 7.0, os principais resultados indicaram que o construto busca de sensação se associou à variação do delinquir, na qual se observou uma associação entre uso potencial de drogas, condutas desviantes e comportamento agressivo.
\end{abstract}

Palavras-chaves: Busca de sensação. Variação do delinquir. Modelo causal. Jovem.

\begin{abstract}
Although there are many variables that explain delinquency of young people, the personality characteristics still have been in Psychology a very promising issue to understand this problem, contributing for an intervention in this problem. In this study we intended to develop, from the modeling of a structural equation, the explanation of the variation of deliquency (related to the potential use of drugs, deviant and aggressive behaviors), based on a personal issue - sensation seeking. 284 youngs, of 14 to 20 years, answered a questionnaire about the potential use of drugs, deviant behaviors, aggressive behavior and sensation seeking. With the software AMOS GRAFICS 7.0, the main results indicated that the question of sensation seeking was associated to the variation of delinquency, in which was observed an association among the potential use of drugs, deviant and aggressive behaviors and aggressive.
\end{abstract}

Keywords: Sensation seeking. Variation of delinquency. Causal model. Adolescent. 


\section{Introdução}

O problema da delinquência entre os jovens não é um fenômeno novo; porém, atualmente, os diversos atos e autores que tem manifestado antissociabilidade ou delito na sociedade vigente tem chamado a atenção de especialistas e leigos. Isso se deve ao fato de que os autores desses problemas, em sua dinâmica social, tangenciam as normas e organização social, causando prejuízo a todos. Trata-se de um fenômeno que tem se destacado porque os jovens que delinquem são aqueles que, supostamente, não apresentam história de delinquência em suas relações interpessoais, familiares ou educacionais, além de possuir boa estrutura social, econômica e escolar. (Stoff, Breiling \& Maser, 1997).

Assim, o problema da delinquência independente da perspectiva teórica sobre o tema ou da interpretação da dinâmica do ato delitivo (Maranhão, 2003; Mira y López, 2009; Neto, 2006; Schneider, 1987; Robert, 2007), sendo definida como um ato juvenil que pretende, de forma premeditada, transgredir as normas socialmente aceitas causando danos físicos e morais às pessoas, bem como prejudicando a sociedade e as relações interpessoais; outra forma da delituosidade caracteriza-se pela organização sócio-grupal adotada pelos jovens com o objetivo de quebrar a norma social (por exemplo, as gangues, a criação de jogos de diversão violentos, balbúrdias em festas, vandalismo, alto consumo de álcool e fumo). Sob essa perspectiva, reflete-se: a delinquência entre os jovens poderia ser compreendida para além dos construtos separados (isto é, o jovem que usa drogas, que tem um comportamento agressivo e tem uma conduta desviante), mas considerando a interdependência entre esses construtos, que abrange de forma mais completa o fenômeno da delinquência.

\section{Um nexo causal da variação do delinquir}

Compreender a dinâmica do delinquir não tem sido apenas uma preocupação de pesquisadores brasileiros: estudos em países da Europa, Oriente, Oceania, América do Norte e América Latina, nos últimos dez anos, têm revelado uma interdependência entre as variáveis que apontam para o binômio "uso de drogas-delinquência", "uso de drogas-violência", "uso de drogas-criminalidade"
- que se influenciam mutuamente (Benvenuti, 2003; D'Andrea, 2008; Frías et al.,-2000; Hein \& Andrade, 2008; Illesca \& Pueyo, 2007). A influência do uso de drogas (lícitas ou ilícitas) se deve ao processo de intoxicação - responsável pela desinibição comportamental, que leva os adolescentes a iniciarem brigas e cometerem furtos ou roubos, quando sob uso frequente de álcool (Osgood, 1998; Parker \& Auerhahn, 1998). Para esses autores, isso independe do que motivou o uso de drogas: seja considerá-las um rito de passagem exigido na fase da adolescência, seja por salientarem uma imagem de poder no grupo ou mesmo por sugestionarem diversão.

Nesse mesmo sentido, estudos de Parker e Auerhahn (1998) sobre criminosos violentos apontam que, em $50 \%$ dos eventos delituosos, havia envolvimento com álcool. 0 uso de drogas e a agressividade configuram um paralelismo, sendo maior a agressividade em pessoas que consomem drogas que em pessoas não consumidoras (Allen, Moeller, Rhoades \& Cherek, 1997; Muntaner et al., 1990). Segundo Negreiros (2001), as pessoas que consumiram drogas quando crianças não apresentavam comportamentos agressivos, mas, quando consumiam ou abusavam de drogas lícitas ou ilícitas em maior idade, o comportamento agressivo emergia. Xue, Zimmerman e Cunningham (2009) concebem que, quando ocorre na adolescência uma iniciação ao uso de álcool, os jovens são mais tarde levados a comportamentos causadores de danos físicos sérios a outras pessoas e à sociedade. Tais comportamentos se manifestam em atos instrumentais hostis em relação às pessoas e objetos (por exemplo, bater, quebrar etc.), por vezes também associados aos seus pares de iguais consumidores de álcool (Xue et al., 2009). Segundo Skara et al. (2008), os comportamentos agressivos são preditos pelo uso e abuso de substâncias entre os jovens; especificamente, os autores observaram que, quanto maior o consumo de álcool, maior a relação com agressão física e pró-social entre os jovens.

No estudo de Young, Sweeting e West (2008), com jovens de 11 a 15 anos, observou-se que, além do álcool provocar desinibição nas relações interpessoais, o seu consumo é também um facilitador para o comportamento antissocial, principalmente em relação à idade; observaram que, quanto maior a díade, mais forte foram os indicadores preditivos entre álcool e conduta antissocial. 0 mesmo observa 
Hemphill, Toumbourou e Catalano (2005) em relação à conduta desviante, compreendida como comportamento antissocial. Esta conduta se inclui na violência e suas formas dissimuladas de agressão, como, por exemplo, a agressão pró-ativa; além disso, esse tipo de conduta entre os jovens é um problema que vai além do prejuízo humano, onerando a economia de municípios e do Estado.

Os estudos de Ferguson, Miguel e Hartley (2009) atribuem uma relação direta entre violência e agressão; não se trata, porém, de uma linearidade causal entre essas variáveis, pois a relação se deve a fatores multideterminantes: associações com pares violentos, estados de depressão, conflitos familiares, distúrbios no humor, relações negativas com adultos (especialmente os adultos responsáveis pelo controle social e comportamental), exposição direta e constante à violência em vídeo games e programas de televisão. Na mesma direção, o estudo de Brook, Whiteman, Balka e Cohen (1997), bem como de Formiga (2010), apontam para a existência de fatores de risco que influenciam as variações da violência (uso de drogas, conduta antissocial e delitiva, agressão); para esses autores, em termos de uma dimensão microssocial, a presença de um vínculo fraco nas interações familiares, a tolerância ao desvio, a intolerância à frustração, um ambiente escolar negativo, além da baixa integração de ego, provavelmente facilitariam a manifestação do fenômeno violento. Assim, essa suposta associação entre drogas, agressão e conduta desviante, permite, no presente estudo, refletir em termos de uma variação da delinquência juvenil.

Desta forma, tem-se como foco a variação do delinquir juvenil, que se refere à capacidade do jovem aderir a um conjunto de condutas potencialmente caracterizadoras do comportamento delinquente; essas condutas podem, de forma direta ou indireta, contribuir para a compreensão da ação ou tendência à delinquência. Esse estudo busca explorar a possibilidade da delinquência juvenil não ser uma manifestação ligada somente ao uso potencial de drogas ou aos comportamentos agressivos e condutas desviantes, mas relacionada a uma interdependência entre esses construtos. A partir da associação entre eles, poder-se-ia destacar que, quanto maior pontuação no uso potencial de drogas, maior a possibilidade do jovem apresentar conduta desviante, bem como comportamentos agressivos - e vice-versa.

\section{Personalidade e variação do delinquir: a busca de sensação como variável preditiva}

Hipotetizada a associação positiva entre o construto uso de drogas, agressão e conduta desviante, atribuída como variação do delinquir juvenil - apesar de terem sido identificadas inúmeras variáveis que explicam o fenômeno da violência em geral (Coelho Junior, 2001; Frías et all., 2000; Formiga et al., 2005; Sobral, 1998) -, espera-se que este modelo teórico seja predito por uma variável personalística: a busca de sensação, intensidade e novidade. Esse modelo tem sido promissor quanto ao poder explicativo das teorias personalísticas, especificamente aquelas que se baseiam na concepção dos traços. Elas têm contribuído para as pesquisas na Psicologia quanto à compreensão do comportamento humano, particularmente em relação aos comportamentos delinquentes (Formiga, 2002; Formiga, 2005; Sobral, 1998; Stephenson, 1990).

A perspectiva dos traços não diz respeito às questões patológicas, mas à díade genética/meio ambiente, implicando no enfoque de que as características individuais consistentes do comportamento, exibido pelo indivíduo em diversas situações, normalmente são concebidas como disposições originadas no processo de socialização (Costa \& McCrae, 1992; John, Donahue \& Kentle, 1991; Saudino \& Plomin, 1996). A partir dessas disposições socializadoras, são geradas taxonomias que permitam ao sujeito se expressar a partir de suas condutas, em formas específicas para si e para os outros quando em interação social (Formiga et al., 2004) - situação que na maioria das vezes atende à desejabilidade social, isto é, o sujeito procura parecer melhor para os outros, descrevendo-se como gostaria de ser descrito por quem o observa, justamente porque essa autoimagem exigida se deve a uma codependência dos "papéis" sociais (Queiroga, Formiga, Jesus, Gouveia \& Andrade, 2001) representados por cada um de nós.

De forma geral, a personalidade tem sido explorada como um construto capaz de explicar as diferenças individuais, proporcionando um importante marco teórico nos estudos a respeito das idiossincrasias do indivíduo e a estabilidade da conduta humana (Ávila, Rodríguez \& Herrero, 1997; Barbaranelli \& Caprara, 1996). Gazzaniga e Heatherton (2005), Paunonen (1998), Peabody (1987) e Trzop (2000), acreditam que, partindo 
desse construto, especificamente, das características individuais, avaliadas cientificamente ou em termos das crenças populares, seja possível, em situações e momentos variados, predizer reações ou disposições futuras das pessoas. 0 conhecimento do construto dos traços de personalidade não somente poderia contribuir para a organização das relações interpessoais, mas também apontar na direção de um fator de proteção nessas relações, principalmente quando se apreende que alguns traços personalísticos são capaz de salientar compormentos pró-sociais ou de desvios (McAdams, 1992).

Partindo do pressuposto de que o jovem tem uma necessidade latente de expandir seu mundo ideal e "real" por meio do comportamento instrumental ou verbal de reivindicação, este se encontra disposto a convites pessoais ou sociais para viver novas descobertas e senti-las intensamente; Formiga, Aguiar e Omar (2008; Omar \& Uribe, 1998) partiram dessa perspectiva teórica, concebendo-a como busca de sensações. Partindo desse construto e dos estudos sobre traços de personalidade e delinquência desenvolvidos por Eysenck (1981), Tranah, Harnett e Yule (1998), Heaven (1996), Ball (2005), Frías et al. (2000), Romero, Luengo e Sobral (2001), Donohew et al. (1990) e Heaven, Newbury e Wilson (2004), percebe-se uma confluência dos seus diferentes instrumentos de análise dos traços de personalidade - especificamente na análise da busca de sensação - comprovando a existência de uma predisposição à conduta desviante juvenil a partir dessa busca.

Diferente do que concebia Zuckerman (1971; Zuckerman, Eysenck \& Eysenck, 1978), e os autores citados no parágrafo anterior, acerca do construto busca de sensação (que pode ser exemplificado pela necessidade de novidades e de viver experiências complexas, motivada apenas pelo desejo de afrontar riscos físicos e sociais, com o intuito de satisfazer necessidades pessoais), Formiga et al. (2008) partiram da perspectiva adotada por Arnett (1994), que defende que esses traços personalísticos estão inseridos na socialização juvenil - compreendendo o comportamento juvenil e, principalmente, aqueles que caracterizam transgressões de normas sociais, como variações do comportamento de risco a partir da investida do jovem em busca de novas experiências e emoções intensas.

Desta forma, neste estudo pretende-se 1) testar um modelo teórico em que o uso potencial de drogas, o comportamento agressivo e as condutas desviantes estejam associados entre si, passando a ser considerados variações do delinquir; 2) explorar a possibilidade de que a busca de sensação (composto pelas dimensões busca de novidade e intensidade) associe-se, positivamente, às variações do delinquir. Para a realização desses objetivos, basear-se-á na modelagem de equação estrutural, considerando-se a conveniência de não se pautar somente os dados obtidos, mas enfatizar um modelo teórico fixo que oriente a extração de indicadores estatísticos entre as variáveis independentes (busca de sensação) e dependentes (variações do delinquir), apresentando qualquer indicação sobre o ajuste dos erros da mensuração no respectivo modelo teórico.

Para que os resultados sejam obtidos, faz-se necessário considerar índices de ajuste (escores covariantes) - destacados na metodologia do presente estudo, na sessão do procedimento -, o que permite enfatizar a teoria e sua explicação, simultaneamente, entre as variáveis independentes e dependentes, além de garantir uma melhor avaliação associativa entre as variáveis a que se pretende corroborar no modelo (Hoe, 2008; Pilati \& Laros, 2007).

Em estudo nessa direção empírica, que tem sido comum na área das ciências humanas e sociais, especificamente na Psicologia (MacCallum \& Austin, 2000; Pilati \& Laros, 2007), partindo do pressuposto teórico, pretende-se, a partir da análise e modelagem de equação estrutural no programa AMOS 7.0, garantir robustez explicativa entre as variáveis, apontando na direção da dinâmica multivariada na verificação da hipótese. Segundo Farias e Santos (2000), por meio desses cálculos espera-se 1) estimativa da magnitude dos efeitos estabelecida entre variáveis, que estão condicionadas ao fato de o modelo especificado (isto é, o diagrama) estar correto, e 2) testar se o modelo é consistente em relação aos dados observados, a partir dos indicadores estatísticos, podendo dizer qual resultado, modelo e dados são plausíveis, embora não se possa afirmar que este seja correto. Atende-se, assim, não a certeza total do modelo, mas a sua probabilidade sistemática na relação entre as variáveis.

Um dos principais objetivos das técnicas multivariadas - neste caso, considera-se a modelagem de equação estrutural - é expandir a habilidade exploratória do pesquisador e a eficiência estatística e teórica no momento de provar a hipótese levantada no estudo. Apesar das técnicas estatísticas 
tradicionais compartilharem limitações, nas quais é possível examinar somente uma relação entre as variáveis, é de suma importância para o pesquisador o fato de ter relações simultâneas; afinal, em alguns modelos existem variáveis que são independentes em algumas relações e dependentes em outras. A fim de suprir esta necessidade, a Modelagem de Equação Estrutural examina uma série de relações de dependência simultâneas, e esse método é particularmente útil quando uma variável dependente se torna independente em relações subsequentes de dependência (Silva, 2006; Hair, Anderson, Tatham \& Black, 2005; Hoe, 2008).

Ao considerar a modelagem estrutural do modelo - isto é, a análise de caminhos (path analysis) relaciona-se as medidas de cada variável conceitual como confiáveis, acreditando não existir erro de medida (mensuração) ou de especificação (operacionalização) das variáveis; cada medida é vista como exata manifestação da variável teórica (Anderson \& Gerbing, 1988; Hair et al., 2005; Zamora \& Lemus, 2008). Assim considerado, desenha-se o modelo teórico que se pretende utilizar, a partir de elaboração hipotética entre as variáveis independente e dependente, isto é, entre as variáveis latentes e variáveis observáveis, por exemplo: no desenho desse modelo - elaboração da ligação entre as figuras caracterizando as variáveis estudadas - um retângulo é considerado como variável observada medida pelo pesquisador; uma elipse é considerada variável latente, isto é, construto hipotético não observado; uma seta com uma ponta indica o caminho ou a relação causal entre duas variáveis; uma seta com duas pontas representa a covariância, isto é, que estas variáveis se associam entre si; por fim, uma bolinha preenchida com um número e letra referem-se a um erro de medida. A partir do momento em que se elabora a hipótese, identifica-se cada uma dessas figuras associando as variáveis que se quer provar a múltipla influência (Silva, 2006; Zamora \& Lemus, 2008).

\section{Método}

Amostra

A amostra foi intencional, sendo composta por 284 jovens, distribuídos igualmente no nível escolar fundamental e médio da rede privada e pública de educação da cidade de João Pessoa - PB, com idades entre 14 e 21 anos. 92\% eram solteiros e possuíam renda econômica media de 2.000,00 reais. Os respondentes foram do gênero masculino e feminino, predominando a participação dos homens (62\%). A decisão por escolher estes participantes se deve ao fato da literatura considerar característico nos sujeitos dessa amostra a manifestação de condutas que configuram o rompimento das normas sociais, ainda que em magnitudes variadas (Pfromm Netto, 1979; Parolin, 2000; Papalia \& Olds, 2000).

\section{Instrumentos}

Inventário de Busca de Sensação: este instrumento, construído por Arnett (1994; Omar \& Uribe, 1998), configura uma escala composta por vinte itens, que por sua vez originam duas subescalas referentes à busca intensidade e novidade na estimulação dos sentidos, cada uma com dez itens. Para respondê-la, a pessoa utilizava uma escala de respostas tipo Likert, com quatro pontos (1 = não me descreve em nada; 2 = descreve-me em alguma medida; 3 = descreve-me bem e 4 = descreve-me totalmente), devendo indicar com um $\mathrm{X}$ ou circular o número que indicasse o quanto cada um dos itens descreve sua conduta habitual.

No Brasil, Formiga e Leime (2011) comprovaram a estrutura fatorial dessa escala. Considerando a análise realizada por Omar e Uribe (1998), verificou-se, com um maior rigor e critério psicométrico, em duas amostras de diferentes estados brasileiros (João Pessoa-PB e Palmas-T0), que o modelo bifatorial, já encontrado, apresentou indicadores psicométricos recomendados na literatura sobre modelagem estrutural, a saber: em João Pessoa - PB: $\chi^{2} / g \mathrm{l}$ $(141,73 / 151)=0,94 ; \mathrm{GFI}=0,94 ; \mathrm{AGFI}=0,92 ; \mathrm{RMR}$ $=0,05 ; \mathrm{NFI}=1,00 ; \mathrm{CFI}=1,00 ; \operatorname{RMSEA}(90 \% \mathrm{IC})=$ $0,00(0,00-0,03)$; $\mathrm{CAIC}=522,84$ e ECVI $=1,11(1,10$ 1,24); e em Palmas - TO: $\chi^{2} / \mathrm{gl}(113,75 / 125)=0,91$; $\mathrm{GFI}=0,96 ; \mathrm{AGFI}=0,94 ; \mathrm{RMR}=0,04 ; \mathrm{NFI}=1,00 ; \mathrm{CFI}$ $=1,00$; RMSEA $(90 \%$ IC) $=0,00(0,00-0,02) ;$ CAIC $=$ 678,38 e ECVI $=1,00(1,00-1,10)$.

POSIT - The Problem Oriented Screening Instrument for Teenagers: validado para o contexto brasileiro por Coelho Junior, Gontiès e Gouveia (2003), trata-se de uma medida correspondente a 81 itens distribuídos em sete fatores que avaliam a potencialidade do futuro consumo de bebidas 
alcoólicas e outras drogas em jovens (por exemplo, seus amigos se aborrecem nas festas onde não servem bebidas alcoólicas?; você causou dano a si mesmo ou a alguém sob o efeito de drogas?; costuma perder atividades ou acontecimentos porque gastou dinheiro com drogas ou bebidas alcoólicas?; sentiu alguma vez que era dependente de álcool ou das drogas?; seus amigos levam drogas, lícitas ou ilícitas, às festas? etc.). Os respondentes deveriam indicar numa escala de resposta de forçada (sim ou não) quanto ao o uso e abuso de substância lícitas e elícitas. Os autores da escala encontraram sete fatores de primeira ordem, que explicaram conjuntamente $27,7 \%$ da variância total e seus índices de consistência interna (Alfas de Cronbach) variaram de 0,65 a 0,80: potencial consumo de álcool e maconha, delinquência juvenil compartilhada, desequilíbrio emocional, conduta antissocial, dificuldades no relacionamento com os pais e/ou responsáveis, dificuldade na aprendizagem, trabalho e desempenho. Estes, além dos alfas de Cronbach estarem entre 0,71 e 0,83 , apresentaram correlações ( $r$ ) positivas e significativas (de 0,27 a 0,51 ) entre os fatores e pontuação total dos itens. Resultados esses que se assemelham aos encontrados por Coelho Junior et al. (2003).

Escala de Condutas Antissociais e Delitivas: este instrumento, proposto por Seisdedos (1988) e validado por Formiga e Gouveia (2003) para o contexto brasileiro, compreende uma medida comportamental em relação às Condutas Antissociais e Delitivas. Tal medida é composta por quarenta elementos, distribuídos em dois fatores, como segue: o primeiro envolve as condutas_antissociais, em que seus elementos não expressam delitos, mas comportamentos que desafiam a ordem social e infringem normas sociais (por exemplo, jogar lixo no chão mesmo quando há perto um cesto de lixo; tocar a campainha na casa de alguém e sair correndo). 0 segundo fator relaciona-se às condutas delitivas. Estas incorporam comportamentos delitivos que estão fora da lei, caracterizando uma infração ou uma conduta faltosa e prejudicial a alguém ou mesmo à sociedade como um todo (por exemplo, roubar objetos dos carros; conseguir dinheiro ameaçando pessoas mais fracas). Para cada elemento, os participantes deveriam indicar o quanto apresentavam o comportamento assinalado no seu dia-a-dia. Para isso, utilizavam uma escala de resposta com dez pontos, tendo os seguintes extremos: $0=$ Nunca e $9=$ Sempre.
A presente escala revelou indicadores psicométricos consistentes, identificando os fatores destacados acima; para a Conduta Antissocial encontrou-se um alfa de Cronbach de 0,86 e para a Conduta Delitiva ou Delinquente, 0,92. Considerando a Análise Fatorial Confirmatória, realizada com o Lisrel 8.0, comprovou-se essas dimensões previamente encontradas $\left(\chi^{2} / \mathrm{gl}=1,35\right.$; AGFI $=0.89$; PHI $(\phi)=0,79, p>0,05)$ na análise dos principais componentes (Formiga \& Gouveia, 2003). Neste estudo, essa escala apresentou alfas próximos aos encontrados nos estudo de Formiga (2003), tanto para Conduta Antisocial (alfa de Cronbach, $\alpha=0,84$ ) e para Conduta Delitiva ou Delinquente, $\alpha=0,90$ mantendo sua consistência interna.

Questionário do comportamento agressivo: este instrumento é composto por 26 itens, distribuídos em quatro fatores: agressão física (por exemplo, "Se alguém me bater, eu bato de volta"), agressão verbal (por exemplo, "eu não consigo ficar calado(a) quando as pessoas discordam de mim"), raiva (por exemplo, "algumas vezes me sinto como uma bomba preste a explodir") e hostilidade (por exemplo, "eu desconfio de pessoas estranhas que são amigáveis demais"). Foi proposto por Buss e Perry (1999) e validado em uma população de infratores por Williams, Boyd, Cascardi e Poythress (1996), encontrando uma estrutura composta por 26 itens distribuídos como agressão física, agressão verbal, raiva e hostilidade. Formiga, Trigueiro, Melo e Dourado (2006), no Brasil, aplicaram essa escala como estudo exploratório numa amostra de jovens não delinquentes, identificando apenas dois fatores, diferentes dos destacados por Buss e Perry (1999); a partir de uma análise exploratória e fatorial confirmatória, identificaram os fatores de agressão física e descompensação emocional, que se relacionavam positivamente.

No presente estudo, realizou-se uma análise fatorial confirmatória (AFC) e de modelagem de equação estrutural (MEE) para este instrumento; este revelou indicadores de ajustes recomendados na literatura vigente (Byrne, 1989; Hair et al., 2005; Van De Vijver \& Leung, 1997): $\chi^{2} / \mathrm{gl}=0,97 ; \mathrm{GFI}=$ 0,96 e AGFI $=0,93 ;$ CFI $=1,00 ; \operatorname{RMSEA}(90 \%$ IC $)=$ $0,00(0,00-0,03)$, CAIC $=851,70$ e ECVI $=1,19$, apresentando garantia de sua confiabilidade fatorial e evidência empírica para aplicação e mensuração no contexto paraibano; esses resultados se diferenciam dos encontrados por Buss e Perry (1999), pois não 
somente a etiquetagem dos fatores estabeleceu-se como agressão física e descompensação emocional, mas a quantidade de itens conglomerados em seus fatores foram menores.

Assim, utilizou-se a adaptação dessa versão no presente estudo, na qual os itens eram respondidos numa escala de resposta do tipo Likert, com os extremos variando de 1 (Discordo Totalmente) a 5 (Concordo Totalmente).

Caracterização Sócio-Demográfica: foram elaboradas perguntas que contribuíram para caracterizar os participantes deste estudo (por exemplo, sexo, idade, classe sócio-econômica), bem como realizar um controle estatístico de algum atributo que possa interferir diretamente nos seus resultados.

\section{Procedimentos}

Todos os procedimentos adotados nesta pesquisa seguiram as orientações previstas na Resolução 196/96 do CNS e na Resolução 016/2000 do Conselho Federal de Psicologia (CNS, 1996; ANPEPP, 2000).

\section{Administração}

Procurou-se definir um procedimento padrão que consistia em aplicar os instrumentos coletivamente em sala de aula, em escolas de diversas áreas urbanas da cidade de João Pessoa - PB. Desta forma, colaboradores com experiência metodológica e ética ficaram responsáveis pela coleta dos dados. Após conseguir a autorização tanto da diretoria da escola quanto dos professores responsáveis pela disciplina, os aplicadores se apresentavam em sala de aula como interessados em conhecer as opiniões e os comportamentos das pessoas em seu cotidiano, solicitando a colaboração voluntária dos estudantes no sentido de responder um breve questionário.

Para isso, foi-lhes dito que não havia resposta certa ou errada e que, mesmo necessitando de uma resposta individual, estes não deveriam sentir-se obrigados a responder o instrumento, podendo desistir a qualquer momento, seja quando tivesse o instrumento em suas mãos, ao iniciar sua leitura ou outra eventual condição. Em qualquer um desses eventos, não haveria problema em sua desistência, apenas bastava contatar as pessoas responsáveis pela aplicação do instrumento na sala de aula. A todos era assegurado o anonimato das suas respostas, enfatizando que elas seriam tratadas, estatisticamente, em seu conjunto de respostas; apesar do questionário ser autoaplicável, contando com as instruções necessárias para que possam ser respondidos, os colaboradores estiveram presentes durante toda a aplicação para retirar eventuais dúvidas ou realizar esclarecimentos que se fizessem indispensáveis, não interferindo na lógica e compreensão das respostas dos respondentes. Um tempo médio de 30 minutos foi suficiente para concluir essa atividade.

\section{Análise dos dados}

No que se refere à análise dos dados desta pesquisa, utilizou-se a versão 15.0 do pacote estatístico SPSS para Windows. Foram computadas estatísticas descritivas (tendência central e dispersão). No AMOS 7.0, foram computados e avaliados os indicadores estatísticos para o Modelo de Equações Estruturais (SEM), considerando, segundo uma bondade de ajuste subjetiva, os seguintes indicadores: $\chi^{2} / g l$ (grau de liberdade), que admite como adequados índices entre 2 e 3, aceitando-se até 5; Raiz Quadrada Média Residual - RMR, indica o ajustamento do modelo teórico aos dados, na medida que a diferença entre os dois se aproxima de zero (0); índices de qualidade de ajuste, dados pelos GFI/ AGFI, que medem a variabilidade explicada pelo modelo, e com índices aceitáveis a partir de 0,80; CFI, que compara de forma geral o modelo estimado e o modelo nulo, considerando valores mais próximos de um (1) como indicadores de ajustamento satisfatório; e o RMSEA, que refere-se ao erro médio aproximado da raiz quadrática e deve apresentar intervalo de confiança como ideal situado entre 0,05 e 0,08 (Byrne, 1989; Hair et al., 2005; Joreskög \& Sörbom, 1989).

\section{Resultados e discussão}

A fim de atender ao objetivo principal do presente estudo - testar o modelo teórico (causal) para explicar as variações do delinquir a partir da busca de sensação - considerou-se um modelo recursivo de equações estruturais. Inicialmente, com o objetivo 
de garantir a perspectiva teórica estabelecida neste estudo sobre a variação do delinquir, hipotetizou-se que o construto uso potencial de drogas associa-se ao comportamento agressivo e à conduta desviante.

Gerou-se no AMOS GRAFICS 7.0 o modelo hipotético proposto, apresentando pesos (saturações) que explicam o modelo em questão, expostos na Figura 1; após as devidas modificações de ajuste do modelo adequado, observou-se uma razão: $\mathrm{c}^{2} / \mathrm{gl}=0,79$; RMR $=0,01 ; \mathrm{GFI}=0,98 ; \mathrm{AGFI}=0,95 ; \mathrm{CFI}=1,00$ e RMSEA $=0,01(0,00-0,04)$. Os pesos da variável uso potencial de drogas associou-se $(\lambda=0,78)$ positivamente à conduta desviante e ao comportamento agressivo $(\lambda$ $=0,65$ ), bem como também se observou uma associação positiva entre essas últimas variáveis $(\lambda=0,16)$ de acordo com o que se hipotetizava (Figura 1).

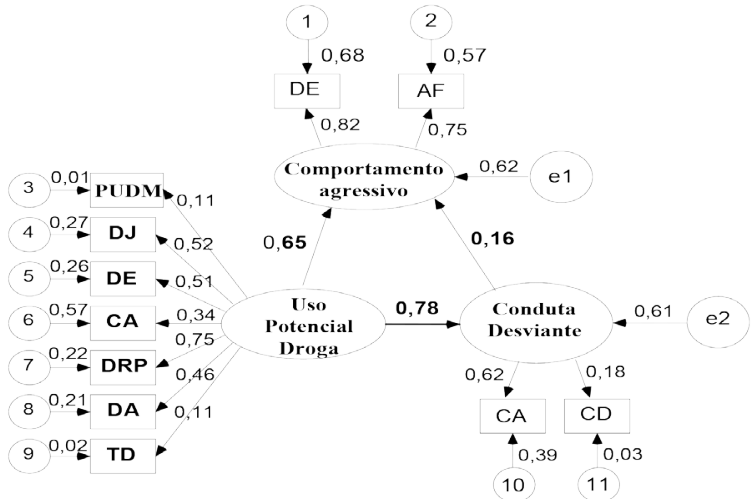

Notas: $\mathrm{DE}=$ Decomposição emocional; $\mathrm{AF}$ = Agressão física; $\mathrm{CA}$ = Conduta antissocial $; \mathrm{CD}=$ Conduta delitiva; $\mathrm{PUMD}=$ Potencial uso de droga e maconha; DJ = Deliquência juvenil; DE = desequilíbrio emocional; DRP = Dificuldade relação parental; $\mathrm{DA}=$ Dificuldade aprendizagem; $\mathrm{TD}=$ Trabalho e desemprego. $x^{2} / \mathrm{gl}=$ 0,$79 ; \mathrm{RMR}=0,01 ; \mathrm{GFI}=0,98 ; \mathrm{AGFI}=0,95 ; \mathrm{CFI}=1,00$ e RMSEA $=0,01(0,00-0,04)$.

Figura 1 - Modelo teórico da explicação entre as variáveis da variação do delinquir juvenil

Comprovada a hipótese estabelecida para o construto variação do delinquir, passou-se a gerar o modelo teórico pretendido sobre a influência dos traços de personalidade (especificamente a busca de sensação) sobre a variação do delinquir (isto é, uso de drogas, comportamento agressivo e conduta desviante). Realizadas as devidas modificações nos parâmetros psicométricos (as covariações entre as variáveis), encontrou-se um modelo adequado para a hipótese esperada, que apresentou a seguinte razão: $\chi^{2} /$ $\operatorname{gl}(5,93 / 7)=0,85, \mathrm{p}<0,55 ; \mathrm{RMR}=0,02 ; \mathrm{GFI}=0,99$; AGFI $=0,97 ;$ CFI $=1,00$ e RMSEA $=0,00(0,00-0,08)$. Observou-se que os pesos da variável considerada busca de sensação - associou-se positivamente $(\lambda=$ 0,38 ) à variação do delinquir, que contempla o uso de drogas, comportamento agressivo e conduta desviante. Todas as saturações (lambdas, $\lambda$ ) foram estatisticamente diferentes de zero $(t>1,96, p<0,05)$, apoiando a adequação do conjunto de variáveis exógenas incluídas no modelo teórico testado.

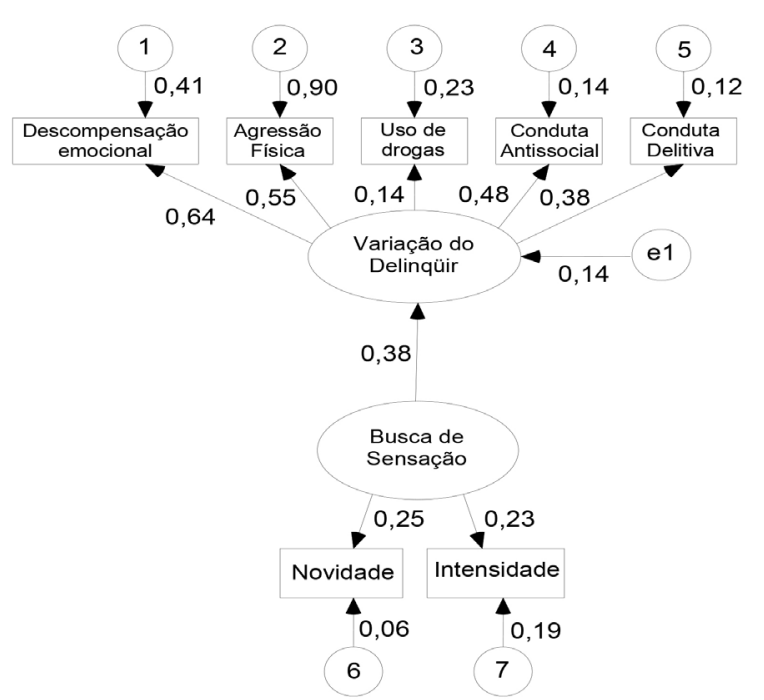

Notas: $x^{2} / \mathrm{gl}=0,79 ; \mathrm{RMR}=0,01 ; \mathrm{GFI}=0,98 ; \mathrm{AGFI}=0,95 ; \mathrm{CFI}=$ 1,00 e RMSEA $=0,01(0,00-0,04)$.

Figura 2 - Modelo teórico da influência da busca de sensação sobre a variação do delinquir juvenil

Comprovado um modelo teórico geral, considerando os construtos latentes da busca de sensação, que apresentou indicadores psicométricos confiáveis, pretendeu-se, a partir desse modelo geral, estabelecer um modelo parcimonioso em que considerasse cada dimensão da busca de sensação como variável observável. Hipotetizou-se um modelo específico em que a busca de sensação a novidade e busca de sensação a intensidade se associe, respectivamente, ao construto variação do delinquir (ver figura 2 ).

A partir de um modelo recursivo, é possível observar na figura 3 , a comprovação dessa nova hipótese esperada. Observou-se um modelo adequado, o qual apresentou a seguinte razão: $\mathrm{c}^{2} / \mathrm{gl}(3,15 / 2)$ $=1,57, \mathrm{p}<0,21 ; \mathrm{RMR}=0,01 ; \mathrm{GFI}=1,00 ; \mathrm{AGFI}=$ 0,95 ; $\mathrm{CFI}=1,00$ e RMSEA $=0,05(0,00-0,15)$. Os pesos da variável independente, busca de sensação a 
novidade, associou-se, positivamente $(\lambda=0,15)$ a variação do delinquir (Figura 3).

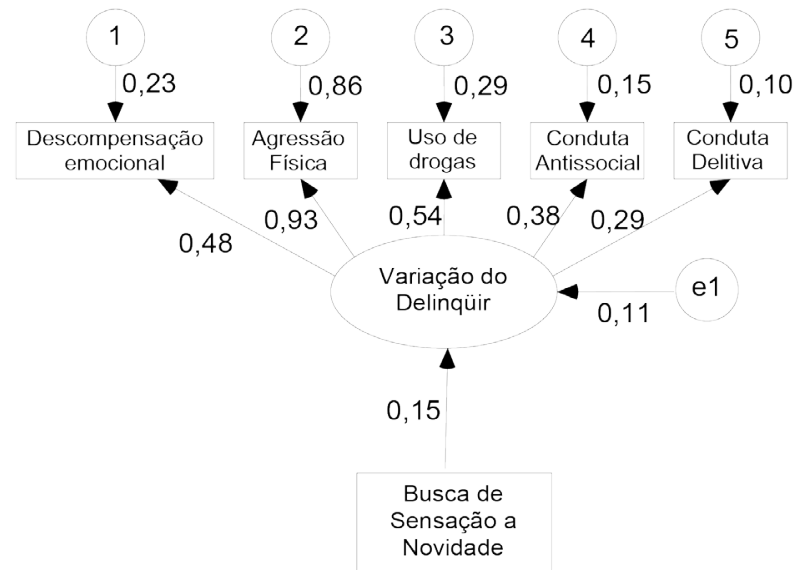

Notas: $x^{2} / \mathrm{gl}=0,71 ; \mathrm{RMR}=0,01 ; \mathrm{GFI}=1,00 ; \mathrm{AGFI}=0,98 ; \mathrm{CFI}=$ 1,00 e RMSEA $=0,05(0,12)$.

Figura 3 - Modelo teórico da influência da busca de sensação a novidade sobre a variação do delinquir juvenil

Seguindo a mesma direção hipotética do modelo anterior, espera-se uma influência da busca de sensação a intensidade sobre a variação do delinquir; na Figura 4 é possível observar a hipótese pretendida. Após as devidas modificações de ajuste comprovou-se um modelo adequado que apresentou a seguinte razão: $\chi^{2} / g l(3,15 / 2)=1,58, \mathrm{p}<0,21$; RMR $=0,01 ; \mathrm{GFI}=0,99 ; \mathrm{AGFI}=0,95 ; \mathrm{CFI}=0,99$ e RMSEA $=0,05(0,00-0,15)$. Observou-se que os pesos da variável independente, busca de sensação a intensidade, associou-se, positivamente $(\lambda=0,34)$ a variação do delinquir (Figura 4).
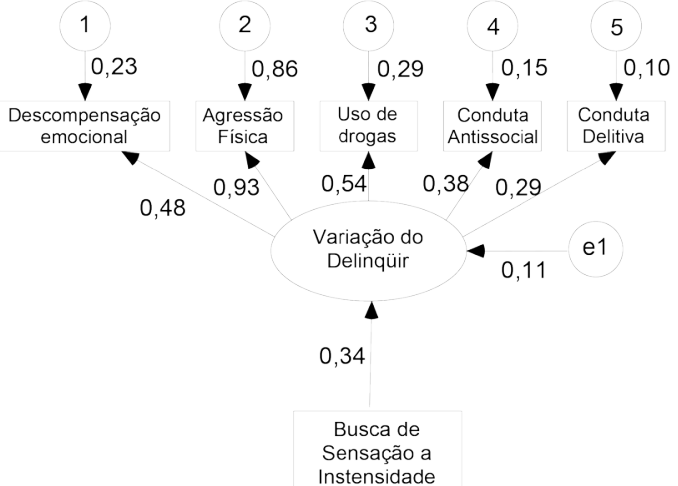

Notas: $x^{2} / \mathrm{gl}=1,57 ; \mathrm{RMR}=0,01 ; \mathrm{GFI}=1,00 ; \mathrm{AGFI}=0,95 ; \mathrm{CFI}=$ 1,00 e RMSEA $=0,05(0,00-0,15)$.

Figura 4 - Modelo teórico da influência da busca de sensação a intensidade sobre a variação do delinquir juvenil
Os resultados aqui apresentados corroboram a hipótese levantada, garantindo a influência da variável busca de sensação sobre variáveis que avaliam o comportamento delinquente. De forma geral, pretendeu-se contribuir, com esses resultados, para o estudo da delinquência; tomando como base explicativa o construto personalístico busca de sensação, aponta-se em direção não somente da perspectiva individual, mas também do processo socializador em que essas diferenças individuais poderão se formar, a fim de compreender os investimentos psicossociais que ocorrem nas relações interpessoais entre os jovens e seu entorno. Essa situação poderá conduzir os jovens para um fator de proteção a partir do momento em que se reconhece o "como, o porquê e o onde" poderá estimular esses jovens a buscarem novas experimentações.

Existem no Brasil estudos que tratam individualmente a relação entre a busca de sensação e cada construto da variação do delinquir; por exemplo, Formiga et al. (2007) observaram a influência da busca de sensação sobre o comportamento agressivo: quanto maior a busca de novidade e intensidade, maior a variação do comportamento agressivo (especificamente agressão verbal, raiva, hostilidade e agressão física). Formiga et al. (2008) observaram que esta variável também influencia a conduta desviante (seja ela antissocial ou delitiva); mas, no presente estudo, observa-se que os resultados desses autores são suficientes para compreender, para montar o "quebra cabeça" da delinquência. Formiga, Omar e Aguiar (2010), além de observarem uma correlação interna entre a busca de sensação a intensidade e novidade, além da relação entre as dimensões que compõem o uso potencial de droga (por exemplo, com o potencial consumo de álcool e maconha, delinquência juvenil compartilhada, desequilíbrio emocional, comportamento antissocial, dificuldades no relacionamento com os pais, dificuldades na aprendizagem e trabalho e desempenho), comprovaram a relação positiva entre busca de sensação e uso potencial de drogas.

0 foco de interesse no presente estudo refere-se ao construto avaliado por Formiga et al. (2007), Formiga et al. (2008) e Formiga et al. (2010), que, apesar de consistente, não é considerado suficiente para explicar o processo da dinâmica delinquente; acredita-se que tais variáveis apresentam, dinamicamente, uma interdependência para o fenômeno da delinquência: o jovem delituoso viola regras na 
sua interpessoalidade social ou individual, esta violação varia entre comportamento agressivo ou conduta desviante, influenciadas pelo uso de drogas lícitas ou ilícitas. Tal reflexão é corroborada na Figura 1, com base nos estudos de Osgood (1998), Parker e Auerhahn (1998), Allen et al. (1997), Muntaner et al. (1990), Negreiros (2001) entre outros autores, já destacados na direção teórica do presente estudo.

Neste contexto, explica-se a variação do delinquir, gerando um sistema teórico do ato delituoso. Sob uma via de mão dupla para compreensão desse problema entre os jovens, a partir desses estudos supracitados, comprovou-se que o problema na delinquência não ocorre no vazio, mas sobre a influência de outros construtos, todos interdependentes: uso potencial de drogas, comportamento agressivo e conduta desviante. Quanto maior o uso potencial de drogas, maior o comportamento agressivo, bem como maior a conduta desviante.

Nessa dinâmica da delituosidade juvenil é possível identificar variáveis individuais que a explicam; neste caso, observando as figuras 2,3 e 4, os traços de personalidade da busca de sensação influenciaram a variação do delinquir (uso de drogas, comportamento agressivo e conduta desviante); especificamente, a busca de novidade e a busca de intensidade revelam, mais ainda, essa influência: tanto o construto - considerado a busca de sensação - quanto as variáveis observadas, a busca de intensidade e novidade, associam-se à variação do delinquir. Ao considerar essas variáveis personalísticas, tais traços não podem se referir à perspectiva patológica, mas chama-se a atenção para a manifestação de traços condutuais, originados dos processos socializadores para construção da personalidade, que são salientes quando relacionados à dinâmica juvenil. Estes traços podem deflagrar uma tendência juvenil ao desvio, na maioria das vezes considerado "coisa de jovem" ou como uma questão que deve ser incluída no "rito de passagem" de ser jovem.

As explicações acerca da variação do delinquir a partir dos traços de personalidade da busca de sensação sugerem focalizar o jovem e sua desorganização emocional, principalmente quanto à construção e organização desses traços de personalidade, que estão inclusos nos processos socializadores da família, da escola e dos pares de iguais (colegas, vizinhos etc.) quando se objetiva o incentivo nos jovens para conhecer pessoas, frequentar lugares para se divertir e sair da monotonia, exigindo uma extroversão e composição de uma maturidade psicológica. Tratase de uma perspectiva situacionista na formação e mudança contextual nos traços de personalidade, tendo a variação da busca de sensação capacidade de predizer o comportamento - neste caso, o comportamento juvenil - quando considerar as disposições individuais e a influência interacional juvenil ao procurar essas experiências novas (Gazzaniga \& Heatherton, 2005; Cloninger, 1999).

\section{Conclusão}

A partir da proposta de Arnett (1994; Omar \& Uribe, 1998), quanto à mensuração da busca de sensação, atenta-se para as investidas tanto da mídia em geral quanto dos pares de iguais e dos familiares em suscitar aos jovens a experiência dos momentos sociais e individuais de sua vida; atenta-se para a experimentação quase que instantânea vivida de forma intensa pelos jovens, que seriam consideradas uma forma de assimilação para a valorização e para a preparação da vida adulta. 0 fato é que, com base nesses resultados, ao instigar uma atitude desse tipo, camufla-se a busca do amadurecimento do desenvolvimento psicológico e social, que somente será possível socializando o jovem em direção às comparações de certo e errado, do respeito e do dever para com os outros; a experiência da busca de sensação, novidade e intensidade não pode deixar de ser sentida e apreendida, mas pode ser vivida não tangenciando as normas sociais e prejudicando as relações interpessoais.

O estudo aponta na direção da adesão do sujeito à busca de sensações, fomentando uma convergência entre as características individuais e o processo socializador vivido pelos jovens. Defende-se que a variação do delinquir provavelmente ocorreria quando o jovem manifestasse maior tendência à busca de novidade e intensidade, seja devido a sua disposição às novas experiências, seja influenciado pelo contexto em que está se socializando. Tendo os resultados confirmados, alguns limites devem ser considerados; a partir desse contexto teórico, reflete-se acerca da situação-disposição no que se refere a predizer o comportamento humano - especificamente a variação do delinquir - com o objetivo de promover fatores de proteção frente ao problema da delinquência juvenil, focalizando principalmente o processo de construção 
dos traços de personalidade; assim, merecem ser destacados: 1) realização de um estudo com essas variáveis enfocando grupos juvenis institucionalizados e não institucionalizados; 2) avaliar, tomando como variáveis de controle o status econômico e a dinâmica familiar, o quanto a busca de sensação explicaria a variação do delinquir; 3) seria também de muito interesse, a partir do ambiente e ideologia escolar, mensurar a busca de sensação e a variação do delinquir; 4) seriam também úteis comparações entre estudos e avaliações clínicas e experimentais em relação às mesmas variáveis do presente estudo.

Considerando os indicadores de bondade de ajuste, provou-se a adequabilidade do modelo, bem como a comprovação da hipótese levantada a partir da perspectiva dos traços de personalidade - especificamente, a busca de sensação. Contudo, assumida a comprovação preditiva das variáveis aqui enfatizadas, é importante destacar que, quando se for considerar os resultados do presente estudo para outros contextos sociais, faz-se necessário, na avaliação dessas variáveis, levar em conta os aspectos mais específicos ou universais entre as culturas (Muenjohn \& Armstrong, 2007; Triandis et al., 1993; Triandis, 1995; Van De Vijver \& Leung, 1997).

\section{Referências}

Allen, T. J., Moeller, F. G., Rhoades, H. M., \& Cherek, D. R. (1997). Subjects with a history of drug dependence are more aggressive than subjects with no drug use history. Drug and Alcohol Dependence, 46, 95-103.

Anderson, J. C., \& Gerbing, D. W. (1988). Structural equation modeling in practice: A review and recommended two-step approach. Psychological Bulletin, 103(3), 411-423.

Associação Nacional de Pesquisa e Pós-Graduação em Psicologia - ANPEPP. (2000). Contribuições para a discussão das Resoluções CNS no. 196/96 e CFP No 016/2000. Recuperado em 2 de set. 2011, de http://www.anpepp.org.br/XIISimposio/Rel_ ComissaoEticasobre_Res_CNS_e_CFP.pdf2000

Arnett, J. (1994). Sensation seeking: a new conceptualization and a new scale. Personality and Individual Differences, 16(2), 289-296.
Ávila, A. E., Rodríguez, S. C., \& Herrero, J. R. S. (1997). Evaluación de la personalidad patológica: Nuevas perspectivas. In E. Cordero (Org.). La evaluación psicológica en el año 2000. (pp. 79-107). Madrid: TEA.

Ball, S. A. (2005). Personality traits, problems, and disorders: Clinical applications to substance use disorders. Journal of Research in Personality, 39, 84-102.

Barbaranelli, C., \& Caprara, G. V. (1996). How many dimensions to describe personality? A comparison of Cattel, Comrey, and the Big Five taxonomies of personality traits. European Review of Applied Psychology, 46(1), 15-24.

Benvenuti, P. (2003). Violencia juvenil y delincuencia en la región de Latinoamérica. Tese em Economia, London School of Economics, Londres.

Brook, J. S., Whiteman, M., Balka, E. B., \& Cohen, P. (1997). Drug use and delinquency: Shared and unshared risk factors in African American and Puerto Rican adolescents. The Journal of Genetic Psychology, 158(1), 25-39.

Buss, A. H., \& Perry, M. (1999). The aggression questionnaire. Journal of Personality and Social Psychology, 63(3), 452-459.

Byrne, B. M. (1989). A primer of LISREL: Basic applications and programming for confirmatory factor analytic models. Nova York: Springer-Verlag.

Cloninger, S. C. (1999). Teorias da personalidade. São Paulo: Martins Fontes.

Coelho Junior, L. L. (2001). Uso potencial de drogas em estudantes do ensino médio: Suas correlações com as prioridades axiológicas. Dissertação de Mestrado em Psicologia Social, Universidade Federal da Paraíba, João Pessoa.

Coelho Junior, L. L., Gontiès, B., \& Gouveia, V. V. (2003). Questionário para detectar potencial uso de drogas entre adolescentes (Posit): Adaptação brasileira. Jornal Brasileiro de Psiquiatria, 52(2), 109-116.

Conselho Nacional de Saúde - CNS. (1996). Diretrizes $e$ normas regulamentadoras de pesquisas envolvendo seres humanos. Recuperado em 2 de set. 2011, de http://conselho.saude.gov.br/resolucoes/reso_96. htm. 1996 
Costa, P. T., \& McCrae, R. R. (1992). Four ways five factors are basic. Personality and Individual Differences, 13, 653-665.

D’Andrea, G. (2008). Delinquência juvenil: A noção de trajetórias desenvolvimentais e a descrição de carreiras. Dissertação de Mestrado em Psicologia, Faculdade de Filosofia, Ciências e Letras de Ribeirão Preto, Universidade de São Paulo, Ribeirão Preto.

Donohew, R. L., Hoyle, R. H., Clayton, R. R., Skinner, W. F., Colon, S. E., \& Rice, R. E. (1990). Sensation seeking and drug use by adolescents and their friends: Models for marijuana and alcohol. Journal of Studies on Alcohol, 60(5), 622-640.

Eysenck, H. J. (1981). Impulsiveness and anti-social behavior in children. Current Psychological Research, 1, 31-37.

Farias, S. A., \& Santos, R. C. (2000). Modelagem de equações estruturais e satisfação do consumidor: uma investigação teórica e prática. Revista de Administração Contemporânea, 4(3), 107-132.

Ferguson, C. J., Miguel, C. S., \& Hartley, R. D. (2009). A multivariate analysis of youth violence and aggression: The influence of family, peers, depression and media violence. Journal of Pediatrics, 155(6), 904-908.

Formiga, N. S. (2002). Condutas anti-sociais e delitivas: Uma explicação baseada nos valores humanos. Dissertação de Mestrado em Psicologia Social, Universidade Federal da Paraíba, João Pessoa.

Formiga, N. S. (2003). Fidedignidade da escala de condutas anti-sociais e delitivas ao contexto brasileiro. Psicologia em Estudo, 8(2), 133-138.

Formiga, N. S., \& Gouveia, V. V. (2003). Adaptação e validação da escala de condutas anti-sociais e delitivas ao contexto brasileiro. Revista Psico, 34(2), 367-388.

Formiga, N. S., Teixeira, J., Curado, F., Fachini, A. C., Yepes, C., \& Omar, A. (2004). Busca de sensações e hábitos de lazer. In 34 Reunião Anual de Psicologia: Formação do psicólogo brasileiro: História de desafios e conquistas. Ribeirão Preto: Sociedade Brasileira de Psicologia.

Formiga, N. S. (2005). Comprovando a hipótese do compromisso convencional: Influência dos pares sócio-normativos sobre as condutas desviantes em jovens. Psicologia: ciência e profissão, 25(4), 602-613.
Formiga, N. S., Saraiva, C. P., Pequeno, N. L. P., Trigueiro, E. S. O., Joca, E. C., \& Leal, R. S. (2005). Diferenças individuais e comportamentos desviantes: Correlatos dos cinco grandes fatores da personalidade e conduta anti-social e delitiva. In Anais do I Congresso LatinoAmericano de Psicologia. São Paulo.

Formiga, N. S., Trigueiro, E. S. O., Melo, C. F., \& Dourado, J. L. G. (2006). A busca de sensação e as variações condutuais da violência juvenil: A experiência em correr risco como explicação das condutas desviantes, comportamento agressivo e uso potencial de drogas. In 58 Reunião Anual da SBPC: Semeando Interdisciplinaridade. Florianópolis.

Formiga, N. S., Cavalcante, C. P., Araújo, T. T. V., Lima, S., \& Santana, R. (2007). Comportamento agressivo e busca de sensação em jovens. Psicologia Argumento, 25(50), 289-302.

Formiga, N. S., Aguiar, M., \& Omar, A. (2008). Busca de sensação e condutas anti-sociais e delitivas em jovens. Psicologia Ciência e Profissão, 28, 668-681.

Formiga, N. S. (2010). Pares sócio-normativos e condutas desviantes: testagem de um modelo teórico. Barbarói, 32(1), 28-43.

Formiga, N. S., Omar, A., \& Aguiar, M. (2010). Busca de sensação e uso potencial de drogas em universitários brasileiros. Psicologia Revista da PUC-SP, 19(2), 97-118.

Formiga, N. S., \& Leime, J. (2011). Inventário de busca de sensação em jovens: Evidência estrutural de uma medida de traços de personalidade. Peritia: Revista portuguesa de psicologia. (Artigo no prelo).

Frías, M. A., Sotomayor, M. P., Varela, C. B. C., Zaragoza, F. O., Banda, A. L. B., \& García, A. S. (2000). Predictores de la delincuencia juvenil. La Psicología Social en México, 8, 486-492.

Gazzaniga, M. S., \& Heatherton, T. F. (2005). Personalidade. In Ciência Psicológica: Mente, cérebro e comportamento. (pp. 470-496). Porto Alegre: Artmed.

Hair, J. F., Tatham, R. L., Anderson, R. E., \& Black, W. (2005). Análise multivariada de dados. Porto Alegre: Bookman.

Heaven, P. C. L. (1996). Personality and self-reported delinquency: Analysis of the "big five" personality dimensions. Personality and individual differences, 20 (1), 47-54. 
Heaven, P. C. L., Newbury, K., \& Wilson, V. (2004). The Eysenck psychoticism dimension and delinquent behaviours among non-criminals: Changes across the lifespan?. Personality and Individual Differences, 36, 1817-1825.

Hein, J., \& Andrade, A. G. (2008). Efeitos do uso do álcool e das drogas ilícitas no comportamento de adolescentes de risco: uma revisão das publicações científicas entre 1997 e 2007. Revista de Psiquiatria Clínica, 35(1), 61-64.

Hemphill, S. A., Toumbourou, J. W., \& Catalano, R. F. (2005). Predictors of violence, antisocial behavior and relational aggression in Australian adolescents: a longitudinal study. Report for the Criminology Research Council.

Hoe, L. S. (2008). Issues and procedures in adopting structural equation modeling technique. Journal of Applied Quantitative Methods, 3(1), 76-83.

Illesca, S. R., \& Pueyo, A. A. (2007). The psychology of criminal conduct. Papeles del Psicólogo, 28(3), 147-156.

John, O. P., Donahue, E. M., \& Kentle, R. L. (1991). The "Big Five" inventory \& versions $4 a$ and 54. University of California, Institute of Personality and Social Research, Berkeley.

Joreskög, K., \& Sörbom, D. (1989). LISREL 7 user's reference guide. Mooresville: Scientific Software.

MacCallum, R. C., \& Austin, J. T. (2000). Applications of structural equation modelling in psychological research. Annual Review of Psychology, 51, 201-226.

Maranhão, O. R. (2003). Psicologia do crime. São Paulo: Malheiros.

McAdams, D. P. (1992). The five-factor personality profiles. Journal of Personality Assessment, 60, 329-361.

Muenjohn, N., \& Armstrong, A. (2007). Transformational leadership: The influence of culture on the leadership behaviours of expatriate managers. International Journal of Business and Information, 2(2), 265-283.

Muntaner, C., Walter, D., Nagoshi, C., Fishbein, D., Haertzen, C. A., \& Jaffe, J. H. (1990). Self-report vs. laboratory measures of aggression as predictors of substance abuse. Drug and Alcohol Dependence, 25, 1-11.

Mira y López, E. (2009). Manual de psicologia jurídica (3aㅡ ed.). São Paulo: Impactus.
Negreiros, J. (2001). Relação entre o consumo de álcool e drogas e comportamentos anti-sociais nos jovens. Toxicodependências, 1, 51-58.

Neto, F. B. (2006). Somos todos criminosos e desonestos? Um estudo sobre a delinquência. Florianópolis: Insular.

Omar, A., \& Uribe, H. D. (1998). Dimensiones de personalidad y búsqueda de sensaciones. Psicologia: Teoria, Investigação e Prática, 3, 257-268.

Osgood, D. W. (1998). Drugs, alcohol and adolescent violence. Center for the Study and Prevention of Violence, University of Colorado Boulder, Boulder. Recuperado em 20 de jun. 2010, de www.colorado.edu/cspv

Papalia, D. A., \& Olds, S. W. (2000). Desenvolvimento humano. Porto Alegre: ArtMed Editora.

Parker, R. N., \& Auerhahn, K. (1998). Alcohol, drugs and violence. Annual Review of Sociology, 24, 291-311.

Parolin, I. C. H. (2000). Adolescência nossa de cada dia: Um trabalho com pais e adolescentes. In Scoz, Lima, B. J. L. et al.. (Orgs.), Psicopedagogia, avanços teóricos e práticos: Escola-família-aprendizagem. (pp. 406412). São Paulo: Vetro.

Paunonen, S. V. (1998). Hierarchical organization of personality and prediction of behavior. Journal of Personality and Social Psychology, 74(2), 538-556.

Peabody, D. (1987). Personality dimensions through trait inferences. Journal of Personality and Social Psychology, 46(2), 384-403.

Pfromm Netto, S. (1979). Psicologia da adolescência. São Paulo: Pioneira.

Pilati, R., \& Laros, J. A. (2007). Modelos de equações estruturais em Psicologia: conceitos e aplicações. Psicologia: Teoria e Pesquisa, 23(2), 205-216.

Queiroga, F., Formiga, N. S., Jesus, G. R., Gouveia, V. V., \& Andrade, J. M. (2001). Desejabilidade social e personalidade. In Anais da XXXI Reunião Anual de Psicologia: A construção da psicologia na pesquisa e no ensino. Rio de Janeiro: SBP.

Robert, P. (2007). Sociologia do crime. Petrópolis: Vozes.

Romero, E., Luengo, M. A., \& Sobral, J. (2001). Personality and antisocial behavior: Study of temperamental dimensions. Personality and Individual Differences, 31, 329-348. 
Saudino, K. J., \& Plomin, R. (1996). Personality and behavior genetics: Where have been and where are we goin?. Journal of Research in Personality, 30, 335-347.

Schneider, L. (1987). Marginalidade e delinquência juvenil. São Paulo: Cortez.

Seisdedos, N. C. (1988). Cuestionario A - D de conductas antisociales - delictivas. Madrid: TEA.

Silva, J. S. F. (2006). Modelagem de equações estruturais: Apresentação de uma metodologia. Dissertação de Mestrado em Engenharia de Produção, Universidade Federal do Rio Grande do Sul, Porto Alegre. Recuperado em 25 de ago. 2009, de http://hdl.handle.net/10183/8628

Skara, S., Pokhrel P., Weiner M. D., Sun, P., Dent, C. W., \& Sussman, S. (2008). Physical and relational aggression as predictors of drug use: gender differences among high school students. Addictive Behaviors, 33(12), 1507-1515.

Sobral, J. (1998). Psicología social jurídica. In J. L. Álvaro, A. Garrido, \& J. R. Torregrosa (Orgs.). Psicología Social Aplicada. (pp. 254-268). Madrid: McGraw Hill.

Stephenson, G. F. (1990). Psicología Social Aplicada. In M. Hewstone, W. Stroebe, J. P. Codol, G. M. Stephenson (Org.). Introducción a la psicología social: Una perspectiva europea. (pp. 397-425). Barcelona: Ariel.

Stoff, D. M., Breiling, J., \& Maser, J. D. (1997). Handbook of Antisocial Behavior. Nova York: John Wiley \& Sons.

Tranah, T., Harnett, P. E., \& Yule, W. (1998). Conduct disorder and personality. Personality and Individual Differences, 24, 741-745.

Triandis, H.C. (1995). Individualism and collectivism. Boulder: Westview Press.
Triandis, H. C., McCusker, C., Betancourt, H., Iwao, S., Leung, K., Salazar, J. M., Setiadi, B., Sinha, B. P., Touzard, H., \& Zaleski, Z. (1993). Na etic-emic analysis of individualism and collectivism. Journal of Cross-Cultural Psychology, 24(3), 366-383.

Trzop, B. M. (2000). The big five: Taxonomy of trait theory. Recuperado em 24 de dez. 2005, de http//www.personality research.org/papers/popkins.html

Van de Vijver, F., \& Leung, K. (1997). Methods and data analysis for cross-cultural research. Thousand Oaks: Sage Publications.

Williams, T. Y., Boyd, J. C., Cascardi, M. A., \& Poythress, N. (1996). Factor structure and convergent validity of the aggression questionnaire in an offender population. Psychological Assessment, 8(4), 398-403.

Xue, Y., Zimmerman, M. A., \& Cunningham. R. (2009). Relationship between alcohol use and violent behavior among urban african american youths from adolescence to emerging adulthood: a longitudinal study. American Journal of Public Health, 99(11), 2041-2048.

Young, R., Sweeting, H., \& West, P. (2008). A longitudinal study of alcohol use and antisocial behaviour in young people. Alcohol and Alcoholism, 43, 204-14.

Zamora, C. S., \& Lemus, I. S. (2008). Modelos de ecuaciones estructurales: ¿qué es eso?. Ciencia \& Trabajo, 10(29), 106-110.

Zuckerman, M. (1971). Dimensions sensation of seeking. Journal of Consulting and Clinical Psychology, 36, 45-52.

Zuckerman, M., Eysenck, S. B. G., \& Eysenck, H. J. (1978). Sensation seeking in England and America: Crosscultural, age and sex comparisons. Journal of Consulting and Clinical Psychology, 46, 139-149. 